\title{
Yeast centromeres coordinate their movements
}

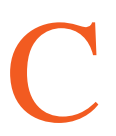

ondensin and cohesin cross-link the pericentromeres of budding yeast chromosomes to coordinate their dynamics during mitosis, Stephens et al. reveal.

In budding yeast, each centromere attaches to a single microtubule during metaphase. The pericentric chromatin surrounding each centromere forms a spring that resists the forces pulling it toward the spindle pole. The resulting tension helps signal that chromosomes are correctly attached to the mitotic spindle and that cells can enter anaphase. Whether each pericentromere forms its own, independent spring or whether the pericentric chromatin of different chromosomes is linked together to coordinate tension across multiple microtubule attachment sites is unclear.

By labeling and live imaging pericentric chromatin, Stephens et al. saw that the pericentromeres of different chromosomes tended to move and stretch together during metaphase, suggesting that they are interlinked. Indeed, the researchers found that different pericentromeres physically associated with one another.

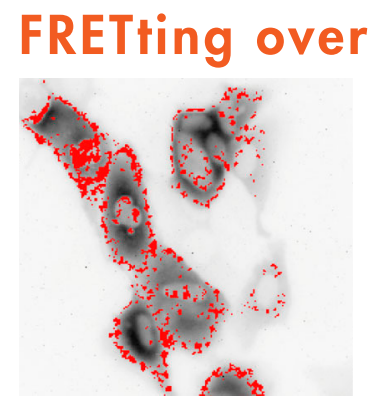

Extended, active KIF 17 (red) localizes to the periphery of MDCK cells.

\section{kinesin activity}

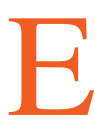
spenel et al. describe how a FRET-based biosensor can be used to track the activity of a kinesin motor protein in living cells.

The kinesin KIF17 promotes the polarization of epithelial cells by stabilizing microtubules, perhaps by coupling their plus ends to the cell cortex. How KIF17's activity is regulated is unknown, however, so Espenel et al. monitored the kinesin's activity in vivo by constructing a version of the protein with GFP and mCherry tags at either end. Inactive KIF17 folds in on itself so that the two fluorescent tags are close enough to undergo Förster resonance energy transfer (FRET). Active KIF17, on the other hand, adopts an extended conformation with the fluorophores Switching on synaptic PP1

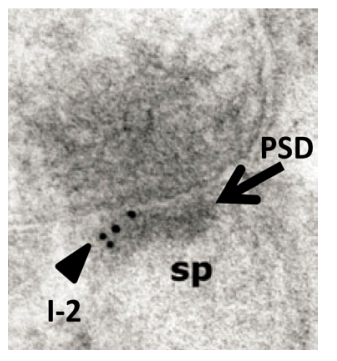

Immunogold EM shows inhibitor-2's localization near the postsynaptic density (PSD) of a dendritic spine (sp).

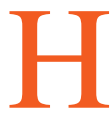

ou et al. describe how neurons activate the protein phosphatase PP1 in response to synaptic stimulation.

PP1 is a key regulator of synaptic plasticity, the phenomenon in which neurons modulate the strength of their synaptic connections in order to store memories. PP1 promotes long-term depression, for example, reducing the function of active synapses by dephosphorylating a number of synaptic and nuclear substrates. But how PP1 is activated during long-term depression is unclear.

PP1 is inhibited by the phosphorylation of a threonine residue in the protein's $\mathrm{C}$ terminus. Hou et al. found that this phosphorylation was reduced in neurons stimulated by the
This association was lost in cells lacking condensin or cohesin, two chromatin-organizing proteins that are enriched on pericentromeres and help form the mitotic chromatin spring. Pericentromeres no longer moved together in cells lacking condensin, and they didn't show coordinated stretching in the absence of cohesin. In addition, the kinesin motor Cin8 helped pericentromeres move and stretch in unison, perhaps by cross-linking microtubules attached to different kinetochores.

Thus, in budding yeast, microtubule attachment sites on different chromosomes are interlinked and coordinated. In mammalian cells, each kinetochore attaches to around 20 dynamic microtubules. Senior author Kerry Bloom thinks that the chromatin surrounding each attachment site may be similarly cross-linked to distribute tension across the kinetochore and stabilize the chromosome's association with the mitotic spindle. He now wants to investigate how pericentric chromatin generates enough force to resist the spindle's pull. Stephens, A.D., et al. 2013. J. Cell Biol. http://dx.doi.org/10.1083/icb.201307104.

too far apart to generate a strong FRET signal.

Espenel et al. used fluorescence lifetime imaging to detect the biosensor's FRET signal and analyzed their data using the "phasor" method so that they could resolve the localization of active and inactive KIF17 molecules within epithelial cells. Active, extended KIF17 concentrated on stable microtubules at the cell periphery. Protein kinase C, part of the microtubule stabilization pathway in other cell types, promoted the kinesin's activation, and the microtubule plus end-tracking protein EB1 recruited active KIF17 to the end of dynamic microtubules to initiate stabilization.

Senior author Geri Kreitzer now wants to follow how KIF17's activity changes as epithelial cells form intercellular adhesions and polarize. Because kinesins control membrane trafficking as well as cytoskeletal dynamics, she also hopes to use similar kinesin biosensors to study many other cellular processes.

Espenel, C., et al. 2013. J. Cell Biol. http://dx.doi.org/10.1083/icb.201305023.

neurotransmitter NMDA. The phosphatase calcineurin and the regulatory protein inhibitor-1 have been proposed to control PP1 activity, but inhibiting these proteins had no effect on PP1 phosphorylation, at least in cortical neurons. Instead, the researchers discovered that the neuron-specific cyclin-dependent kinase Cdk5 phosphorylates PP1's C terminus. NMDA stimulation induced the degradation of Cdk5's activator p35, limiting the kinase's activity so that PP1 was able to dephosphorylate and activate itself.

NMDA also triggered the dephosphorylation of a phosphatase regulator called inhibitor-2, boosting the protein's association with PP1. Surprisingly, knocking down inhibitor-2 enhanced the inhibitory phosphorylation of PP1 and prevented the induction of long-term depression. This suggests that, despite its name, inhibitor- 2 may promote PP1 activity in neurons. Senior author Houhui Xia now wants to examine the effect of depleting inhibitor-2 in vivo and to determine how the protein affects PP1's activity toward different neuronal substrates.

Hou, H., et al. 2013. J. Cell Biol. http://dx.doi.org/10.1083/jcb.201303035. 